\title{
Ingestión de lípidos oxidados: efecto sobre actividad enzimática antioxidativa en trucha arcoiris Oncorhynchus mykiss (Walbaum)
}

\section{Oxidized lipids intake: effect on antioxidative enzimatic activity in rainbow trout Oncorhynchus mykiss (Walbaum)}

\author{
Jorge Zambrano N, ${ }^{1 *}$ Zoot, Miguel Landines P, ${ }^{1}$ Ph.D.
}

\begin{abstract}
${ }^{1}$ Universidad Nacional de Colombia, Facultad de Medicina Veterinaria y de Zootecnia, Laboratorio de Fisiología de Peces. Bogotá, Colombia. *Correspondencia: jazambranon@unal.edu.co.
\end{abstract}

Recibido: Junio de 2010: Aceptado: Agosto de 2011.

\section{RESUMEN}

Objetivo. Evaluar el efecto de la ingestión de lípidos durante períodos cortos (20 días) y largos (90 días) sobre la actividad hepática y en tracto gastrointestinal (TGI) de las enzimas catalasa (CAT), superóxido dismutasa (SOD) y glutatión peroxidasa (GPX). Materiales y métodos. Se utilizó el Índice peróxidos (VP) y el índice anisidina (VA) para detectar la presencia de productos de la oxidación en las raciones. Se realizó un análisis de varianza bajo un modelo de parcelas divididas en el tiempo. Cuando se encontraron diferencias $(p<0.05)$ las medias fueron comparadas mediante la prueba de Tukey $(5 \%)$. Resultados. Las raciones presentaron altos niveles de oxidación durante todo el experimento y hubo diferencias significativas entre tratamientos. La actividad SOD presentó niveles decrecientes a nivel hepático durante los dos períodos de exposición, sin embargo, en TGI se generó un incremento significativo de actividad SOD (175\%) en individuos sometidos a todos los tratamientos. La actividad CAT presentó un alto nivel de correlación con la actividad SOD en todos los períodos de exposición y órganos. La actividad GPx presentó diferencias significativas para los dos períodos de exposición en hígado y al día 90 en TGI, indicando alta sensibilidad de la enzima ante la ingestión de peróxidos. Conclusiones. La actividad GPx en TGI mostró coeficientes de correlación superiores a 0.95 , sugiriendo que es buen indicador del estado oxidativo de las raciones.

Palabras clave: Aceites de pescado, catalasa, glutatión peroxidasa, superóxido dismutasa, oxidación (Fuente: $C A B$ ). 


\section{ABSTRACT}

Objective. To determine the effect, of different oxidation level of lipid, ingested for a period of 20 and 90 days on Catalase (CAT), Superoxide dismutase (SOD) and Glutathione peroxidase (GPX) activities in hepatical and gastrointestinal tissues. Materials and methods. Peroxide Value (PV) and Anisidine Value (AV) were used to detect products of lipidic oxidation in feeds. Data were analyzed using an split plot design and analysis of variance (ANOVA). Means were compared with Tukey test (5\%). Results. All rations offered showed high peroxides and secondary products of oxidation levels during all assay and in most cases there were significant differences of oxidative quality between dietary treatments $(p \leq 0.05)$. SOD activity showed decreasing levels in liver for both exposure periods, however, in GIT was generated a significant increase in SOD activity (175\%) across time in the individuals subjected to all treatments. CAT activity showed high correlation level with SOD activity for the two periods of exposure and in both organs.

Conclusions. GPx activity showed differences for both exposition periods in liver and at 90 day in GIT, indicating high sensitivity of the enzyme to peroxides intake. In all cases were high correlation coefficients (>0.95), suggesting that GIT-GPx activity is a good indicator of the lipid oxidative status of rations.

Key words: Catalase, fish oils, glutathione peroxidase, superoxide dismutase, oxidation, (Source: $C A B)$.

\section{INTRODUCCIÓN}

La acuicultura es el sector productivo de mayor crecimiento en las últimas décadas a nivel mundial y en Colombia (1). Durante los últimos años se han alcanzado notables desarrollos en los campos biotecnológico, reproductivo, nutricional y de manejo acuícola que han permitido la expansión y consolidación de esta actividad a nivel nacional.

La trucha arcoíris (Oncorhynchus mykiss) es un salmónido originario de los tributarios del río Sacramento en California, Norteamérica; su crecimiento óptimo en sistemas de producción de carne se da entre 13 y $18^{\circ} \mathrm{C}$. Ocupa el tercer lugar entre las especies piscícolas cultivadas en Colombia (1), contribuye con el $1 \%$ de la producción global acuícola total y con el $1.7 \%$ de la producción global en aguas continentales (2). Esta especie requiere altos niveles de inclusión de aceite de pescado en las raciones para satisfacer sus requerimientos de ácidos grasos poli-insaturados de la serie omega 3 (AGPI n-3). Actualmente la industria productora de alimentos balanceados para trucha arcoíris maneja niveles de inclusión de lípidos cercanos al $15 \%$ con el objetivo de mejorar las tasas de conversión alimenticia (FCA) e incrementar las concentraciones de AGPI n-3 en los filetes (3). La inclusión de altos niveles de lípidos en las raciones puede afectar la salud del pez, generando reducción de las tasas de crecimiento debido al deterioro oxidativo de los lípidos que conlleva a la formación de compuestos impalatables con acción citotóxica y teratogénica (4). A pesar de ello, los organismos acuáticos y terrestres cuentan con una serie de mecanismos celulares detoxificantes que contribuyen a la reducción de los daños ocasionados por estos compuestos. Enzimas antioxidantes como la glutatión peroxidasa (GPx), la catalasa (CAT) y la superoxido dismutasa (SOD) constituyen la defensa primaria contra el daño celular oxidativo (5).

El objetivo del presente estudio fue evaluar el efecto de la ingestión de aceite de pescado con distintos grados de oxidación sobre la actividad de Catalasa (CAT), Superóxido dismutasa (SOD) y Glutatión peroxidasa (GPX) a nivel hepático y en el tracto gastrointestinal de juveniles de trucha arcoíris (Oncorhynchus mykiss [Walbaum]). 


\section{MATERIALES Y MÉTODOS}

Sitio de estudio. La fase de campo se desarrolló en las instalaciones de Truchas de la Sierra Ltda. Bogotá, Colombia, bajo condiciones ambientales adecuadas para adelantar el cultivo de trucha arcoíris en fases iníciales de desarrollo ( $T$ promedio: $\left.10^{\circ} \mathrm{C}, \mathrm{pH}: 7.0, \mathrm{O} 2:>7.5 \mathrm{ppm}\right) . \quad \mathrm{El}$ alimento experimental fue desarrollado en el laboratorio de nutrición de peces de la Facultad de Medicina Veterinaria y de Zootecnia (FMVZ) de la Universidad Nacional de Colombia sede Bogotá. Los análisis de calidad oxidativa del aceite de pescado experimental y la determinación de la actividad de las enzimas fueron realizados en los laboratorios de Toxicología y Toxicología Acuática de la FMVZ.

Elaboración del alimento experimental. Se formuló un alimento balanceado a través del software UFFDA ${ }^{\circledR}$ indicado para la fase de levante de trucha arcoíris. Mediante análisis proximal realizado en el laboratorio de nutrición de la FMVZ, de acuerdo con los métodos oficiales de análisis de la asociación de química analítica (6), se comprobó que el alimento contenía $43 \%$ de proteína cruda, $22 \%$ de extracto etéreo, $12 \%$ de humedad, $4 \%$ de fibra y $11 \%$ de cenizas.

Materias primas. Se obtuvieron en una planta productora de alimento comercial para trucha. El alimento fue elaborado en las instalaciones del laboratorio de nutrición de peces de la FMVZ, mediante el empleo de una extrusora experimental EXMICRO ${ }^{\circledR}$ (Exteec máquinas). En su elaboración y almacenamiento no hubo adición de la fracción lipídica aportada por el aceite de pescado. Su composición se muestra en la tabla 1.

Se utilizó una premezcla vitamínica libre de vitamina $E$ y una premezcla mineral libre de selenio, con el fin de observar el comportamiento dinámico de la curva de producción de compuestos primarios y secundarios de la oxidación para asociarlo con la respuesta fisiológica de los peces. La adición de los nutrientes omitidos habría desestimulado la aparición de productos de la oxidación lipídica en el alimento y a
Tabla 1. Materias primas utilizadas para la elaboración del alimento.

\begin{tabular}{|c|c|c|c|c|c|}
\hline \multirow{2}{*}{ Materia prima } & \multicolumn{5}{|c|}{ Nivel de inclusión $(\mathbf{g} / \mathbf{K g})$} \\
\hline & T1 & T2 & T3 & T4 & T5 \\
\hline $\begin{array}{l}\text { Harina de sangre tipo } \\
\text { Spray }\end{array}$ & 50 & 50 & 50 & 50 & 50 \\
\hline Torta de Soya 48 & 160 & 160 & 160 & 160 & 160 \\
\hline $\begin{array}{l}\text { Harina de pescado } \\
\text { Anchoveta ( } 59 \% \text { PC) }\end{array}$ & 400 & 400 & 400 & 400 & 400 \\
\hline Harina de trigo & 148.6 & 148.6 & 148.6 & 148.6 & 148.6 \\
\hline Salvado de trigo & 0.5 & 0.5 & 0.5 & 0.5 & 0.5 \\
\hline $\begin{array}{l}\text { Harina de vísceras } \\
\text { aves }\end{array}$ & 79.8 & 79.8 & 79.8 & 79.8 & 79.8 \\
\hline $\begin{array}{l}\text { Cloruro de Colina } \\
(70 \%)\end{array}$ & 0.1 & 0.1 & 0.1 & 0.1 & 0.1 \\
\hline $\begin{array}{l}\text { Premezcla vitamínica } \\
\text { (Libre de Vit E) }\end{array}$ & 3 & 3 & 3 & 3 & 3 \\
\hline $\begin{array}{l}\text { Premezcla mineral } \\
\text { (Libre de Selenio) }\end{array}$ & 3 & 3 & 3 & 3 & 3 \\
\hline $\begin{array}{l}\text { Aceite subproductos de } \\
\text { tilapia FRESCO }\end{array}$ & 140 & 42 & 70 & 98 & 0 \\
\hline $\begin{array}{l}\text { Aceite subproductos de } \\
\text { tilapia OXIDADO }\end{array}$ & 0 & 98 & 70 & 42 & 140 \\
\hline $\begin{array}{l}\text { Aceite de pescado } \\
\text { marino }\end{array}$ & 15 & 15 & 15 & 15 & 15 \\
\hline TOTAL & 1000 & 1000 & 1000 & 1000 & 1000 \\
\hline
\end{tabular}

su vez mitigado el efecto de estos sobre el sistema de enzimático antioxidativo hepático e intestinal.

Preparación y adición de aceites experimentales. Del total del extracto etéreo contenido en el alimento utilizado en campo (22\%), $15.4 \%$ correspondió al aporte del aceite de pescado experimental y el restante $6.6 \%$ fue aportado por las demás materias primas empleadas en la formulación.

Los componentes de la fracción lipídica fueron integrados por i) Aceite de subproductos de tilapia fresco, ii) Aceite de subproductos de tilapia sometido a un proceso de inducción oxidativa y iii) Aceite comercial de origen marino. Este último tipo de aceite se empleó para asegurar el aporte mínimo de ácidos grasos del tipo omega 3 que no alcanzaba a aportarse con el subproducto de la tilapia.

Al inicio del estudio, se realizó un análisis de contenido de ácidos grasos de los aceites experimentales (proveniente de subproductos de tilapia y el de origen marino), con el objetivo de tipificarlos y de ajustar los niveles ofertados de 
ácidos grasos poliinsaturados a los requerimientos de la especie. Al aceite de subproductos de tilapia no se le había adicionado antioxidantes, mientras que al aceite de origen marino sí (como parte del esquema comercial de manejo en la planta productora de concentrado).

El aceite de subproductos de tilapia fue obtenido 30 días antes del inicio del experimento, se le evaluó el estado oxidativo (índice de peróxidos y valor de anisidina), se separó en dos fracciones del mismo lote: una de ellas fue inmediatamente almacenada en refrigeración durante la totalidad del tiempo del experimento, mientras la otra fue sometida durante 25 días (200 hr) a un proceso de inyección de aire, calentamiento y aporte lumínico. El aceite de pescado de origen marino fue muestreado para la evaluación del estado oxidativo e inmediatamente llevado a refrigeración.

A partir de las materias primas lipídicas mencionadas se diseñaron los tratamientos experimentales (Tabla 2).

Tabla 2. Tratamientos dietarios experimentales.

\begin{tabular}{lccc}
\hline & \multicolumn{3}{c}{$\begin{array}{c}\text { Nivel de inclusión en la fracción lipídica } \\
\text { experimental (\%) }\end{array}$} \\
Tratamiento & $\begin{array}{c}\text { Aceite } \\
\text { subproductos } \\
\text { de tilapia } \\
\text { oxidado }\end{array}$ & $\begin{array}{c}\text { Aceite } \\
\text { subproductos } \\
\text { de tilapia } \\
\text { fresco }\end{array}$ & $\begin{array}{c}\text { Aceite de } \\
\text { pescado } \\
\text { de origen } \\
\text { marino }\end{array}$ \\
\hline $\begin{array}{l}\text { Tratamiento } \\
\text { (Control +) }\end{array}$ & 0.0 & 91.0 & 9.0 \\
Tratamiento 2 & 27.0 & 64.0 & 9.0 \\
Tratamiento 3 & 45.5 & 45.5 & 9.0 \\
Tratamiento 4 & 64.0 & 27.0 & 9.0 \\
Tratamiento 5 & 91.0 & 0.0 & 9.0 \\
(Control -) & & & \\
\hline
\end{tabular}

Periódicamente (cada 21 días), se realizó una nueva mezcla de los aceites experimentales (en las proporciones definidas) con las cantidades a utilizar de alimento almacenado. Esta estrategia se implementó con el fin de realizar las mediciones del estado oxidativo directamente en la materia prima lipídica para eliminar los efectos que tienen los métodos de extracción de lípidos (7) sobre la adecuada determinación de la calidad oxidativa de la fracción lipídica.
Animales. Se seleccionó un grupo homogéneo de 160 juveniles de trucha arcoiris (Oncorhynchus mykiss $[$ Walbaum $])(\mathrm{CV}=3.55 \%, \mathrm{DS}=0.5214)$ de aproximadamente 15 centímetros de longitud (37 g), los cuales fueron distribuidos aleatoriamente 5 tratamientos con 4 réplicas cada uno, para conformar 20 grupos de 8 individuos. Luego fueron ubicados en veinte jaulas de 117 litros de capacidad efectiva cada una suspendidas en estanques asegurando condiciones normales de recambio $(>5 / \mathrm{hr}$ ) y densidad poblacional final $\left(10 \mathrm{Kg} / \mathrm{m}^{3}\right)$.

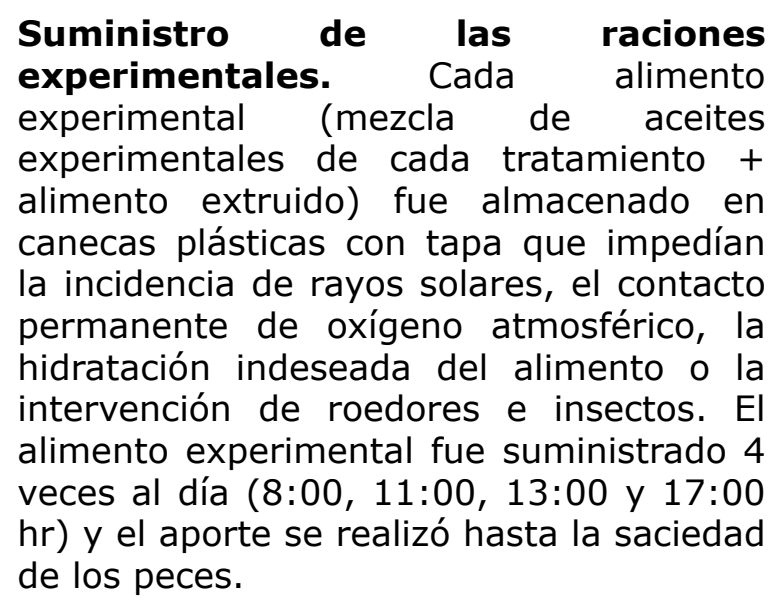

Evaluación oxidativa de las raciones experimentales. Se evaluó la producción de compuestos primarios (peróxidos e hidroperóxidos) y secundarios (aldehídos) de la oxidación lipídica del aceite de pescado experimental mediante las pruebas de índice de peróxido (VP) e índice de anisidina (VA) descritas en las Normas Técnicas Colombianas 236 y 4197, respectivamente $(8,9)$. Estas evaluaciones se realizaron cada 21 días para cada tratamiento (correspondiente a la cantidad de sesiones de remezcla de aceites experimentales para cada tratamiento con el alimento extruido). Este procedimiento se desarrolló para tratar de minimizar el efecto de "almacenamiento del alimento en granja" sobre el desarrollo de productos oxidativos y para obtener un mayor grado de correspondencia entre los niveles declarados de VP y VA en laboratorio con los aportes reales de dichos compuestos a los animales experimentales en granja (a partir del momento en que se realizaba la mezcla 
de aceites con el alimento y se sacaban las muestras de análisis, iniciaba un proceso de diferenciación de comportamientos en términos de evolución del estatus oxidativo entre las muestras estudiadas contra el alimento experimental de aporte en la granja).

Con los valores VP y VA generados para cada tratamiento se construyó un indicador único y global del estatus oxidativo de las raciones experimentales o Índice total de oxidación (TotOX) que correspondía a la asociación de los valores obtenidos así:

TotOX $=2 \mathrm{VP}+\mathrm{VA}$

Estos procedimientos se realizaron al lote "madre" de aceite (30 días antes del inicio del estudio), en el momento de la primera preparación de alimentos experimentales y en las siguientes jornadas de mezcla de alimento (días 21, 42, 63 y 84) por duplicado.

\section{Respuesta animal. Proceso de} sacrificio y extracción de órganos. Los días 20 y 90 se realizó la extracción aleatoria de 2 individuos por jaula ( 8 por tratamiento/sesión, 40 total/sesión) para extraer el tracto gastrointestinal (TGI) y el hígado. Se registró la longitud total, la jaula de proveniencia y realizó la insensibilización mediante transección de la espina dorsal, luego se expusieron los órganos y se muestrearon los tejidos de interés. Los órganos fueron lavados individualmente con solución salina helada al $0.9 \%$ y empacados en frascos marcados que contenían la misma solución a $0^{\circ} \mathrm{C}$.

Transporte de órganos y proceso de homogenización. Los órganos fueron transportados (1.5 hr) en una cava de poliuretano en hielo seco. En el laboratorio se procedió a extraer cada órgano, secarlo, registrar su peso y se realizó la homogenización de órganos $(10,11)$, empleando una solución de buffer fosfato de sodio $0.1 \mathrm{M} \mathrm{pH} 7.4+\mathrm{KCl}(1.17 \%)$ (dilución 1:9 hígado:buffer, dilución 1:5 TGI:buffer). Para la homogenización del tejido hepático se emplearon homogenizadores manuales tipo Potter Everhelm mientras que para la homogenización del TGI se empleó un homogenizador eléctrico de tejidos (Motor: CAT X120, Rotor - generator : Cole palmer 36900-61). Luego se Centrifugó a 12500 RPM durante 30 minutos a $4^{\circ} \mathrm{C}$ para obtener fracción S9 (Heraeus Labofuge 400R). El sobrenadante fue dividido en 4 crioviales para determinar SOD, GPx, CAT y proteína. Estos fueron marcados y almacenados a $-80^{\circ} \mathrm{C}$ hasta su análisis.

\section{Determinación y análisis de la actividad enzimática antioxidativa en hígado y} TGI. Se evaluó la actividad de las enzimas CAT, GPx y SOD en hígado y en TGI mediante espectrofotometría (Shimadzu UV - 160A), para cada individuo y por duplicado. Para el caso de GPx, se midió la oxidación de NADPH a $340 \mathrm{~nm}$, definiendo su actividad en función de nmol de NADPH oxidados/minuto/mg de GPx. Para CAT, la medición se realizó a 240 $\mathrm{nm}$ y se definió la actividad como nmol de $\mathrm{H}_{2} \mathrm{O}_{2}$ consumidos/minuto/mg de CAT (12). La actividad de SOD se midió a $550 \mathrm{~nm}$; se definió como la cantidad de SOD necesaria para producir un $50 \%$ de inhibición de la tasa de reducción del ferricitocromo C (13). Para cada período de exposición al agente estresor (día 20 y día 90) se muestrearon 8 animales por tratamiento dietario. Se evalúo la actividad de cada enzima (CAT, SOD y GPX) por duplicado en el hígado y el tracto gastrointestinal.

Se empleó el método del ácido bicinconínico (Pierce Chemical Company, Rockford, IL, EUA) para estimar la concentración de proteína de las muestras. Se determinó el nivel de proteína de cada órgano proveniente de los peces sometidos a cada tratamiento dietario (160 muestras).

Análisis estadístico. Los datos del nivel de peróxidos (VP), anisidina (VA) y el índice de oxidación total (TotOX) de las diferentes raciones experimentales y los datos de actividad de SOD, GPx y CAT fueron igualmente sometidos a un análisis de varianza de dos vías con un arreglo de parcelas divididas en el tiempo. Se empleó una prueba de Tukey $(p<0.05)$ para detectar diferencias significativas entre tratamientos. En todos los análisis se empleó el paquete estadístico SAS (14). 


\section{RESULTADOS}

Calidad lipídica de las raciones. Se detectó un alto nivel de oxidación principalmente generado por la producción de compuestos secundarios de la oxidación lipídica que permitía tipificarlo como un aceite altamente oxidado. Los valores de VP, VA y TotOX para cada día de evaluación $[21,42,63$ y 84$]$ se presentan en las figuras 1,2 y 3 , respectivamente.

El análisis realizado el día 21 mostró que existieron diferencias significativas entre los tratamientos dietarios $(p=0.0004)$. T1 presentó los niveles de VP más altos, en contraste, los niveles más altos de VA fueron exhibidos por T5. VP y VA mostraron un coeficiente de correlación de -0.99. El índice de oxidación total (TotOX) tuvo un comportamiento similar a VP.

Al día 42, los tratamientos T1, T2 y T3 presentaron niveles deVPsignificativamente superiores a T4 y T5, los niveles de VA fueron ligeramente superiores a los hallados el día 21. Al día 63 se encontró la misma tendencia que al día 21; sin embargo, los niveles de VP fueron 5 veces superiores. No se encontraron diferencias significativas entre tratamientos al calcular el TotOX.

Al día 84 decrecieron significativamente los niveles de peróxidos (VP) y el índice de anisidina (VA) en todos los tratamientos, no se hallaron diferencias significativas entre tratamientos para las variables VP ni TotOx.

\section{Actividad enzimática antioxidativa. Superóxido dismutasa (SOD). La} actividad SOD a nivel hepático no presentó diferencias significativas entre tratamientos dietarios para ninguno de los dos períodos de exposición, sin embargo, al comparar la actividad SOD hepática promedio en los dos períodos de exposición (Corto, día 20 y largo, día 90) se encontró que la actividad de la enzima decreció $29 \%$ en promedio al día 90 con respecto a la observada inicialmente. En contraste, a pesar de que no se hallaron diferencias significativas al medir la actividad SOD en TGI dentro de cada período de evaluación, se observó que la actividad al día 90 fue 2 veces en promedio superior a la observada al día 20 (Figura 4).

Catalasa (CAT). Para la actividad CAT a nivel hepático no se hallaron diferencias significativas entre grupos sometidos a tratamientos dietarios para ninguno de los dos períodos de exposición (Figura 5). Sin embargo, en TGI al día 20 mostró diferencias significativas, encontrándose la mayor actividad en peces alimentados con el tratamiento dietario T5, seguido por T3 y T4. Al día 90 se redujo dicha diferencia. No obstante, se encontró que la actividad en TGI fue en promedio 2.75 veces superior a la observada al día 20. Por el contrario, la actividad CAT a nivel hepático presentó niveles tendientes a la baja ( $87 \%$ en promedio) con respecto a la actividad promedio observada al día 20 .

Glutatión peroxidasa (GPx). La actividad GPx a nivel hepático presentó diferencias significativas entre tratamientos para los dos períodos de exposición. Al día 20, la menor actividad de la enzima fue mostrada por los peces sometidos al tratamiento control ( $T 1$ ), mientras que la mayor actividad fue exhibida por T2. Al día 90 aumentó la diferencia entre respuestas, encontrándose alta actividad en T5 que fue significativamente más alta que en los tratamientos T2, T3 y T1, respectivamente (Figura 6 ). En TGI no se presentaron diferencias significativas al día 20 de exposición. Sin embargo, al día 90 se presentaron diferencias significativas $(p=0.002)$ entre todos los tratamientos. En términos generales la actividad GPx promedio se incrementó con el tiempo en cerca de $70 \%$ a nivel hepático y $82 \%$ en TGI al comparar los dos períodos de exposición.

Correlación calidad del alimento vs. actividad enzimática. Se calculó el grado de correlación de los niveles de compuestos primarios (VP), secundarios (VA) y el índice total de oxidación (TOtOX) al día 21 y 84 con la actividad enzimática antioxidativa CAT, SOD y GPx al día 20 y 90. Para los niveles de VP, VA y TotOX no se encontró una respuesta altamente correlacionada en la actividad CAT a nivel hepático ni en TGI para ninguno de los dos períodos de medición. Similar respuesta fue obtenida para la actividad SOD. 


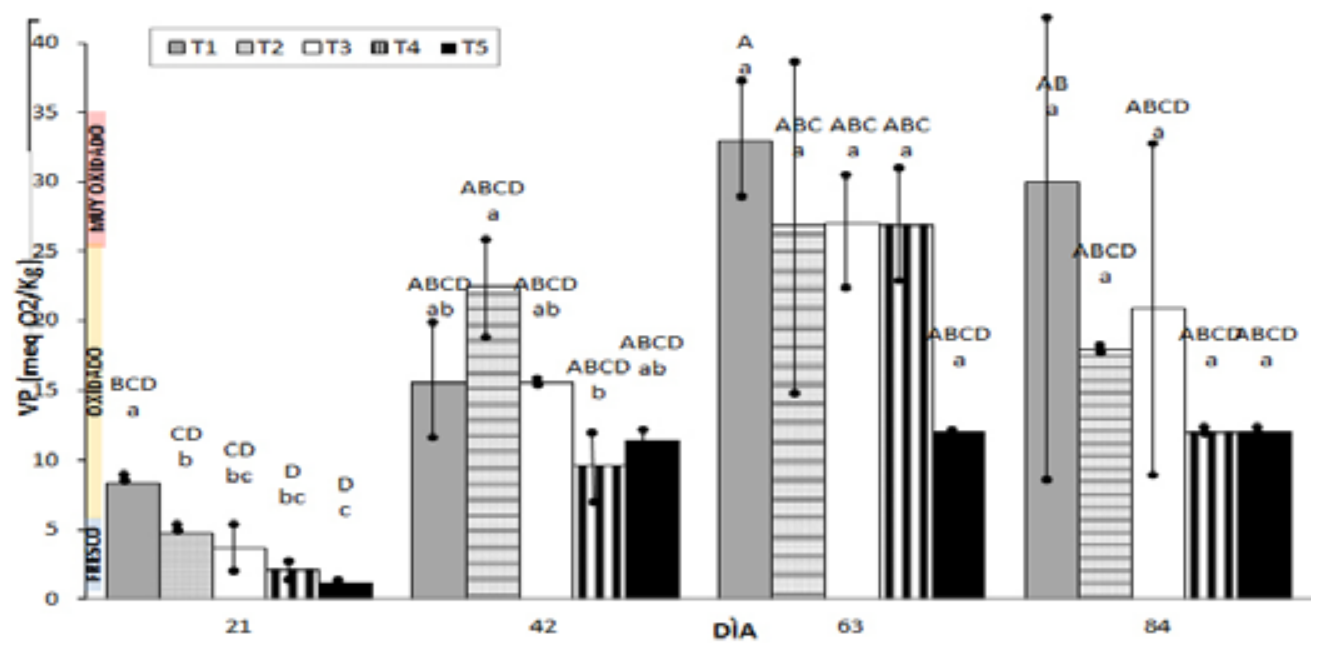

Figura 1. Valores medios \pm D.S. de valor de peróxidos. Letras mayúsculas diferentes indican diferencia significativa $(p<0.05)$ entre tratamientos. Letras minúsculas diferentes indican diferencia significativa $(p<0.05)$ entre jornadas de medición.

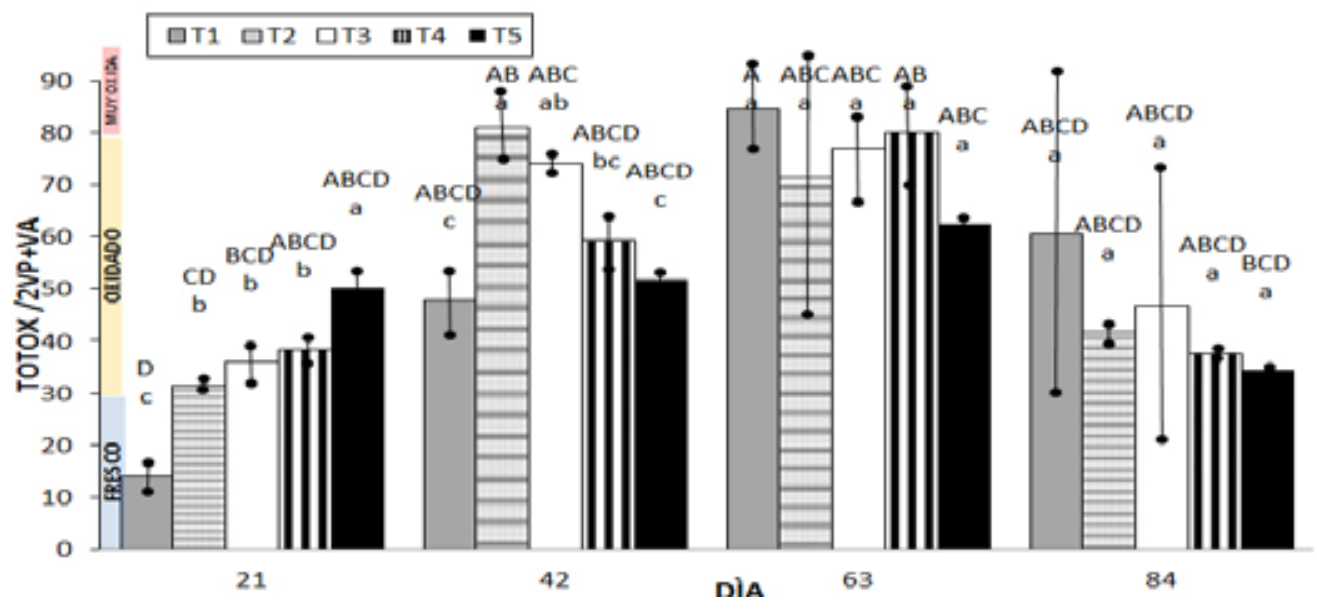

Figura 2. Valores medios \pm D. S. de Valor de Anisidina. Letras mayúsculas diferentes indican diferencia significativa $(p<0.05)$ entre tratamientos. Letras minúsculas diferentes indican diferencia significativa $(p<0.05)$ entre jornadas de medición.

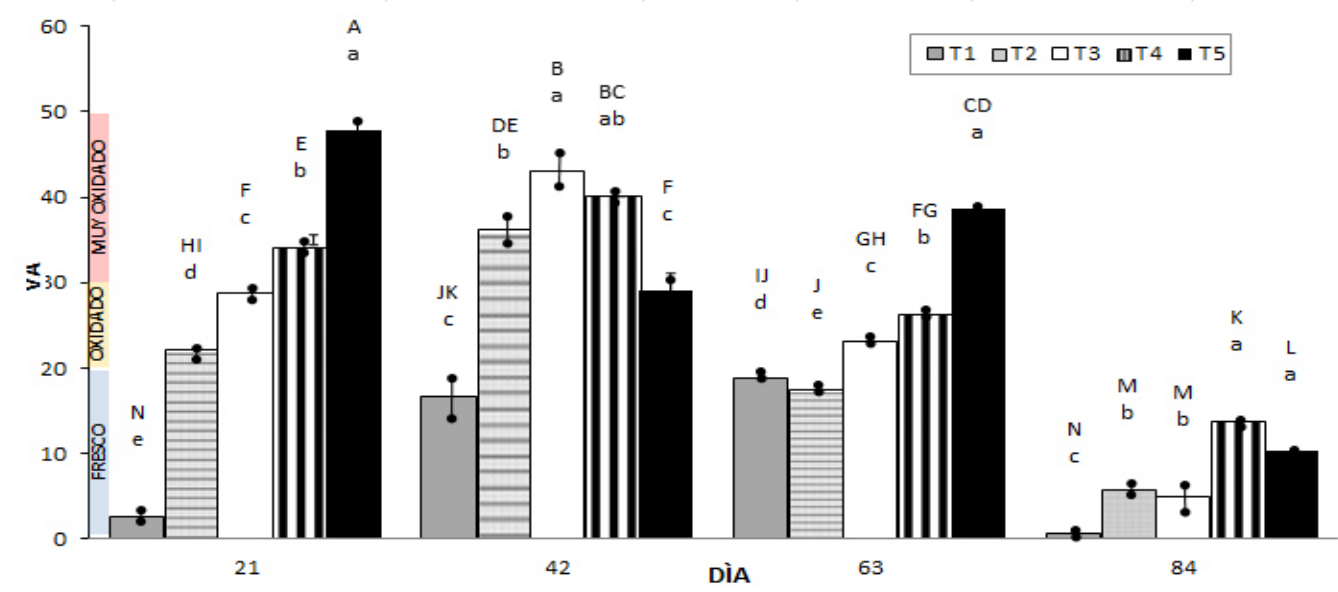

Figura 3. Valores medios \pm D. S. del índice de oxidación total TotOX. Letras mayúsculas diferentes indican diferencia significativa $(p<0.05)$ entre tratamientos. Letras minúsculas diferentes indican diferencia significativa $(p<0.05)$ entre jornadas de medición. 

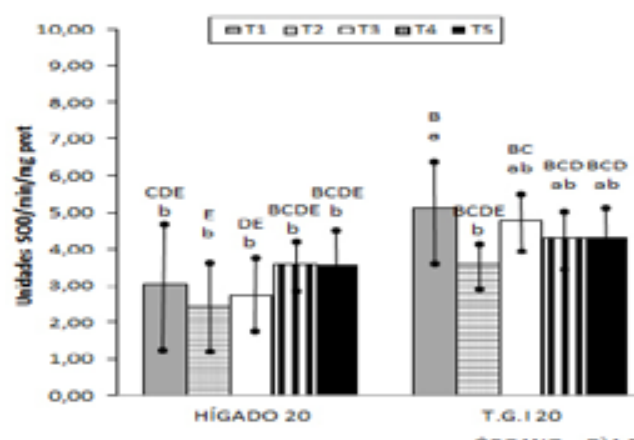

ORGANO - O
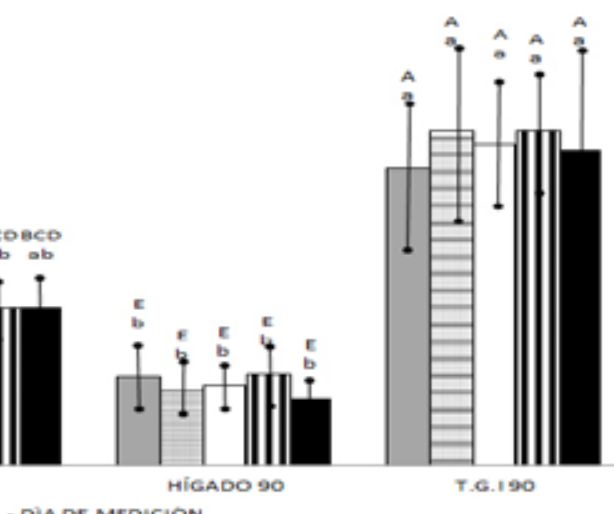

Figura 4. Valores medios \pm D. S. de la actividad de la enzima Superóxido Dismutasa (SOD) a nivel hepático e intestinal a corto (día 20) y largo (Día 90) períodos de exposición. Letras mayúsculas diferentes indican diferencia significativa $(p<0.05)$ entre actividades SOD medidas en órganos a distintos días de exposición a tratamientos dietarios. Letras minúsculas diferentes indican diferencia significativa $(p<0.05)$ entre actividades SOD evaluadas para cada órgano durante cada tiempo de exposición a tratamiento dietarios.

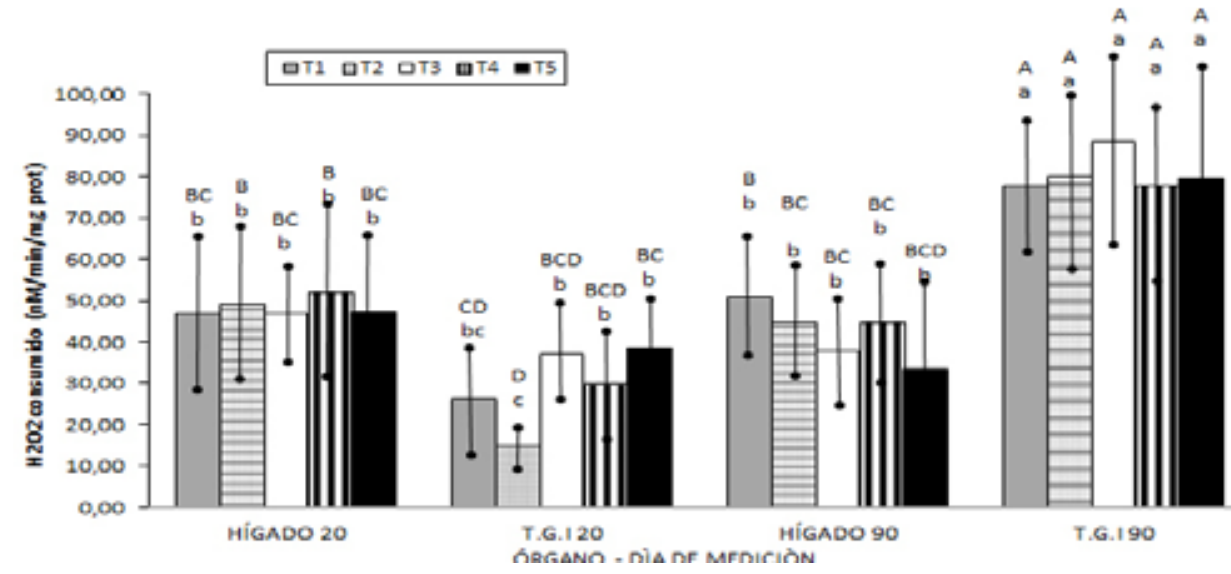

Figura 5. Valores medios \pm D. S. de la actividad de la enzima Catalasa (CAT) a nivel hepático e intestinal a corto (día 20) y largo (Día 90) períodos de exposición. Letras mayúsculas diferentes indican diferencia significativa $(p<0.05)$ entre actividades CAT medidas en órganos a distintos días de exposición a tratamientos dietarios. Letras minúsculas diferentes indican diferencia significativa $(p<0.05)$ entre actividades CAT evaluadas para cada órgano durante cada tiempo de exposición a tratamiento dietarios.

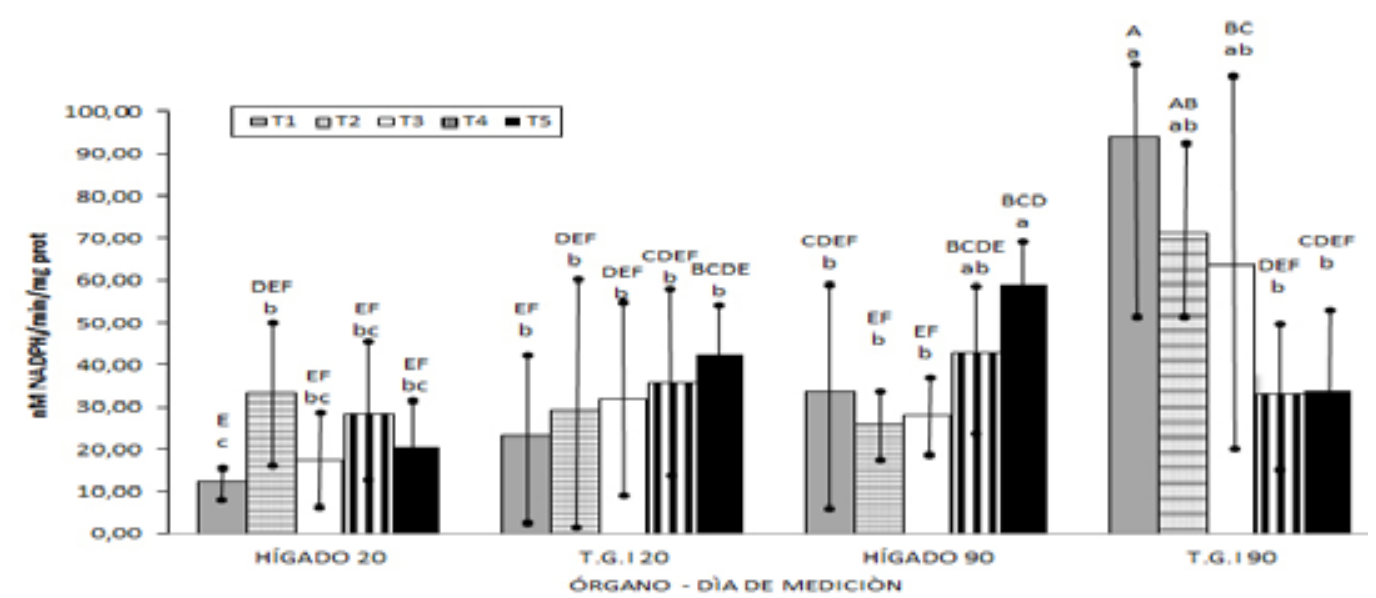

Figura 6. Valores medios \pm D. S. de la actividad de la enzima glutatión peroxidasa (GPx) a nivel hepático e intestinal a corto (día 20) y largo (Día 90) períodos de exposición. Letras mayúsculas diferentes indican diferencia significativa $(p<0.05)$ entre actividades GPx medidas en órganos a distintos días de exposición a tratamientos dietarios. Letras minúsculas diferentes indican diferencia significativa $(p<0.05)$ entre actividades GPX evaluadas para cada órgano durante cada tiempo de exposición a tratamiento dietarios. 
Por otro lado, la actividad GPx en TGI al día 20 mostró niveles de alta correlación con VP, VA y TotOX $(-0.95 ; 0.98$ y 0.97 , respectivamente). Para el día 90 se encontraron niveles de correlación igualmente altos $(0.95 ;-0.96$ y 0.91 , respectivamente). A nivel hepático no se halló alta correlación entre los niveles de peróxidos y aldehídos con la actividad GPx.

\section{DISCUSIÓN}

Calidad lipídica de las raciones. A lo largo de todo el experimento el aceite de pescado de origen marino presentó altos niveles de aldehídos detectados por la prueba de anisidina. A pesar de haber sido obtenido en una planta comercial de producción de alimento balanceado, el aceite fue catalogado como aceite muy oxidado de acuerdo con la escala valorativa de Masson (15). Esto pudo haber sido causado por [i], la no adición de antioxidante o la adición de una cantidad inferior a la requerida para evitar los procesos auto-oxidativos o porque [ii] el aceite contaba con antioxidante, pero este fue adicionado después de las fases de iniciación y propagación de la autooxidación lipídica. Esto sugiere que es necesario implementar las técnicas de evaluación del estado oxidativo que detecten productos primarios y secundarios de la autooxidación. Actualmente, la industria de análisis de alimentos y fabricación de balanceados para animales no emplea técnicas para la determinación de productos secundarios de la oxidación.

Todos los tratamientos dietarios presentaron altos niveles iníciales de anisidina y TotOX (día 21) que excedieron los valores establecidos por los estándares de calidad para aceites frescos (VP entre 3.9 y 5 meq $\mathrm{O}_{2} / \mathrm{kg}$, VA de 10 a 20 y TotOX de 17.8 a 30) y que lo tipificaba como aceite oxidado (VP entre 7.0 y 26 meq $\mathrm{O}_{2} / \mathrm{kg}$, VA de 25 a 30 y TotOX de 39.0 a 82.0) (15) (Figuras 1,2 y 3). Durante la fase inicial de alimentación predominaron los altos niveles de productos secundarios de la oxidación (aldehídos volátiles), por lo que se considera que tales compuestos pudieron haber generado altas mortalidades (>30\%) para el día 20.
Para el día 42, la mayor parte de los tratamientos dietarios alcanzaron niveles de aceite oxidado (15) (Figuras 1, 2 y 3), lo que probablemente pudo incrementar la tasa de mortalidad promedio (hasta $40 \%$ ). Para el día 63, los niveles de peróxidos (VP) en las raciones experimentales (a excepción de T5) se incrementaron hasta alcanzar la tipificación de aceite muy oxidado (VP >26 meq $\mathrm{O}_{2} / \mathrm{kg}$ ) (15) (Figura 1), sin embargo, los niveles de VA se mantuvieron en todos los tratamientos dietarios (a excepción de T5 que presentó un alto nivel de VA que permitió catalogarlo como aceite muy oxidado para esta variable) (Figura 2). Todos los tratamientos dietarios presentaron índices de oxidación total (TotOX) que los clasificaron como raciones que contenían aceite oxidado (Figura 3 ).

Al día 84, se encontró que no existían diferencias significativas entre tratamientos al evaluar VP y calcular TotOX, sin embargo, se encontraron diferencias significativas entre los niveles de VA (Figura 2). En general para este período final, los niveles de VA, VP y TotOX descendieron, permitiendo tipificar las raciones como oxidadas.

\section{Actividad enzimática antioxidativa. Actividad Superóxido Dismutasa (SOD). La enzima SOD transforma el anión superóxido $\mathrm{O}_{-2}$ (Producto de la respiración celular) en $\mathrm{H}_{2} \mathrm{O}_{2}$. Múltiples trabajos han reportado alto nivel de correlación entre la actividad SOD y el metabolismo oxidativo general, lo que indica que los niveles de SOD se ajustan a los niveles de fuentes endógenas de especies oxígeno reactivas (16).}

A pesar de que no se encontraron diferencias significativas entre tratamientos en ninguno de los dos períodos de exposición, los niveles de actividad SOD en TGI se incrementaron dramáticamente del día 20 al día 90 (175\% en promedio). Sin embargo, a nivel hepático la actividad de la enzima decreció $13 \%$ en promedio al día 90 con respecto a los niveles de actividad hallados al día 20 de exposición. Estos hallazgos sugieren que el TGI se convirtió a lo largo del ensayo en el órgano con mayor metabolismo oxidativo posiblemente 
debido al contacto mecánico directo con el agente estresor oxidativo (16).

LOS niveles promedio de actividad SOD a nivel hepático en el presente ensayo $(2.6 \mathrm{U} /$ $\mathrm{min} / \mathrm{mg}$ proteína) fueron muy similares a los hallados en 0 . mykiss ( $2.5 \mathrm{U} / \mathrm{min} / \mathrm{mg}$ proteína) (16), Sparus aurata (5.2 U/min/mg proteína) (17), Penaeus vannamei $(1.72 \mathrm{U} / \mathrm{min} / \mathrm{mg}$ proteína)(18), Nacella concinna $(3.0 \mathrm{U} / \mathrm{min} / \mathrm{mg}$ proteína)(19) y en Scophthalmus maximus, Hippoglossus hippoglossus y S. aurata (4.5; 5.7 y $5.2 \mathrm{U} / \mathrm{min} / \mathrm{mg}$ proteína, respectivamente) (5), pero bastante inferiores a los hallados en Dentex dentex (150 U/min/mg proteína)(20) y en 0 . mykiss (835 U/min/mg proteína)(13).

Actividad catalasa (CAT). La actividad CAT es el indicador principal de la tasa de metabolización del peróxido de hidrógeno $\left(\mathrm{H}_{2} \mathrm{O}_{2}\right)$ para evitar sus efectos nocivos a nivel celular. La actividad CAT es específica para peróxido de hidrógeno $\left(\mathrm{H}_{2} \mathrm{O}_{2}\right)$. Al evaluar el efecto de cada tratamiento dietario para el día 20 en la actividad CAT en TGI se encontraron diferencias significativas entre tratamientos dietarios. Sin embargo, al día 90 a pesar del fuerte incremento en la actividad CAT-TGI no se hallaron diferencias de respuesta entre tratamientos dietarios. La actividad CAT en TGI sufrió un incremento dramático (275\%) desde el día 20 al día 90 de medición. Al día 20 se presentó un nivel promedio de actividad de $29.52 \mathrm{nmol} \quad \mathrm{H}_{2} \mathrm{O}_{2} / \mathrm{min} / \mathrm{mg}$ proteína, que está muy cerca de lo observado (31.64 nmol $\mathrm{H}_{2} \mathrm{O}_{2} / \mathrm{min} / \mathrm{mg}$ proteína) en la misma especie, el mismo órgano y bajo condiciones normales de alimentación (13). Sin embargo, tras 90 días de exposición al agente estresor oxidativo dietario la actividad CAT-TGI se incrementó hasta llegar a 80.90 nmol $\mathrm{H}_{2} \mathrm{O}_{2} / \mathrm{min} / \mathrm{mg}$ proteína en promedio. Este incremento coincide con la tendencia incremental en la producción de compuestos primarios y secundarios de la oxidación lipídica (Figuras 1, 2 y 3).

En el presente trabajo, la actividad CAT a nivel hepático fue en promedio (48.62 y $42.5 \mathrm{nmol} \mathrm{H}_{2} \mathrm{O}_{2} / \mathrm{min} / \mathrm{mg}$ proteína al día 20 y 90 , respectivamente) superior a los niveles encontrados en O. mykiss ( $32 \mathrm{nmol} \mathrm{H}_{2} \mathrm{O}_{2} /$ $\mathrm{min} / \mathrm{mg}$ proteína)(16), pero inferior a los reportados para la misma especie $(116 \mathrm{nmol}$
$\mathrm{H}_{2} \mathrm{O}_{2} / \mathrm{min} / \mathrm{mg}$ proteína)(13). A nivel hepático se han reportado actividades similares de CAT en otras especies: $10.6 \mathrm{nmol} \mathrm{H}_{2} \mathrm{O}_{2} / \mathrm{min} /$ mg proteína en Colossoma macropomum (21), $24.77 \mathrm{nmol} \mathrm{H}_{2} \mathrm{O}_{2} / \mathrm{min} / \mathrm{mg}$ proteína en Sander vitreus (22), $160 \mathrm{nmol} \mathrm{H}_{2} \mathrm{O}_{2} / \mathrm{min} /$ mg proteína en Acipenser naccarii (13) y $180 \mathrm{nmol} \mathrm{H}_{2} \mathrm{O}_{2} / \mathrm{min} / \mathrm{mg}$ proteína en Channa punctatus (12).

La disminución observada en la actividad CAT a nivel hepático, evidencia un proceso de adaptación al estrés generado por la ingestión de peróxidos. El proceso adaptativo estuvo posiblemente sustentado por el incremento sistemático de la actividad CAT en TGI, lo que sugiere que la acción antioxidativa CAT se fue desplazando al órgano de contacto directo con el agente estresor dietario para tratar de evitar la acción hepatotóxica del $\mathrm{H}_{2} \mathrm{O}_{2}$.

De acuerdo con lo observado en $D$. dentex (20), el incremento en la actividad de CAT encontrado en TGI al comparar el día 20 y 90 de exposición y el decrecimiento de la actividad de la misma enzima a nivel hepático son directamente proporcionales con la actividad SOD en los mismos órganos para los mismos períodos de exposición. Esto es debido a que, como enzimas componentes de un sistema de detoxificación oxidativa celular, los niveles de $\mathrm{H}_{2} \mathrm{O}_{2}$ generados por la actividad SOD estimularon niveles proporcionales de actividad CAT.

Actividad glutatión peroxidasa (GPx). A pesar que la actividad GPx también está involucrada en la remoción de $\mathrm{H}_{2} \mathrm{O}_{2}$, la respuesta diferencial en la actividad GPx y CAT (también evidenciada en el presente estudio) indica que existen mecanismos de regulación diferentes para las dos enzimas. GPx está mayoritariamente involucrada en la remoción de peróxidos orgánicos (20).

La actividad GPx presentó diferencias significativas a nivel hepático para los días 20 y 90 de exposición y en TGI al día 90. Al realizar un análisis de correlación de la actividad GPx a nivel hepático y en TGI con VP, VA y TotOX se encontró que a nivel hepático los niveles de correlación eran inferiores a 0.5. Sin embargo, en TGI 
excedieron 0.95 para todos los períodos de exposición, indicando que el TGI fue el órgano de contacto directo con los agentes estresores oxidativos dietarios en el que se incrementó el metabolismo antioxidativo a través del incremento en la actividad GPx.

Los niveles promedio de actividad GPx a nivel hepático al día 20 y $90(21.97$ y $38.34 \mathrm{nmol} \mathrm{NADPH}$ consumidos $/ \mathrm{min} / \mathrm{mg}$ de proteína, respectivamente) estuvieron por debajo de los hallados en hígado de S. aurata alimentado con lípidos oxidados durante 30 y 60 días (72 y 55 nmoles NADPH consumidos/min/mg de proteína, respectivamente)(17). En ese experimento, los niveles de GPx ascendieron a través del tiempo debido a que probablemente se contaba con la acción antioxidativa de la vitamina E. Concordantemente, se han reportado niveles hepáticos de actividad GPx de 66.79 nmoles NADPH consumidos/ $\mathrm{min} / \mathrm{mg}$ de proteína en O. mykiss, bajo condiciones normales de alimentación y sin agentes estresores externos aparentes (13). En otro estudio (23) se reportaron niveles de actividad GPx hepática de 70 nmoles NADPH consumidos/min/mg de proteína en esta misma especie bajo condiciones de realimentación luego de un período de restricción alimenticia de 70 días de duración. Los niveles de actividad GPx en hígado en nuestro ensayo son semejantes a los reportados en $S$. vitreus (29.06 nmol NADPH consumidos/min/mg de proteína)(22).

Los niveles promedio de actividad GPx en TGI al día 20 y 90 (33.10 y 59.95 nmol NADPH consumido/min/mg de proteína, respectivamente) fueron muy inferiores a los hallados en TGI de la misma especie (667.3 nmol NADPH consumidos/min/mg de proteína) bajo condiciones normales de alimentación y sin agentes estresores oxidativos externos aparentes (13).
Los resultados indicaron que los efectos de la ingestión de aceite oxidado sobre la actividad GPx están basados en el rol de la enzima dentro del sistema de detoxificación oxidativa celular y conforme a lo esperado estuvo influenciado por el contenido de peróxidos dietarios, lo mismo ha sido hallado y concluido en otros estudios (17).

En conclusión, se puede afirmar que las raciones ofertadas a lo largo del experimento presentaron siempre altos niveles de productos primarios y secundarios de la oxidación lipídica lo que permitió clasificarlas como "muy oxidadas". Las actividades SOD y CAT fueron directamente proporcionales a lo largo del experimento demostrando el alto nivel de complementariedad de las dos enzimas dentro del sistema de detoxificación oxidativo celular. Estas actividades (SOD y CAT) a nivel hepático decrecieron a lo largo del experimento, sin embargo se incrementaron en TGI, lo que sugiere un proceso de adaptación al estrés oxidativo. Por otro lado, la actividad GPx se incrementó a nivel hepático y en TGI a lo largo del período de exposición al estrés oxidativo, indicando la alta resiliencia de la enzima a estímulos oxidativos dietarios. La actividad GPx en TGI estuvo altamente correlacionada (>0.95) con la calidad oxidativa de las raciones aportadas a lo largo del experimento. Por lo tanto y de acuerdo con las evidencias, si se quiere determinar el impacto de la ingestión de raciones oxidadas en trucha arcoíris, es recomendable evaluar la actividad GPx en TGI.

En términos generales, los niveles actividad fueron (en la mayoría de los casos) más altos en TGI que en hígado y la actividad promedio para todas las enzimas fue más alta al día 90 que al día 20. 


\section{REFERENCIAS}

1. Espinal F, Martínez H, González F. La cadena de la piscicultura en Colombia. Una mirada global en su estructura y dinámica 1991 - 2005. [En línea]. Ministerio de Agricultura y desarrollo rural. Observatorio Agrocadenas de Colombia. 2005; [Accesado 22 Jul 2010]. URL disponible en: http://www. agronet.gov.co/www/docs_agronet/ 2005/112164315_caracterizacion_ piscicultura.pdf.

2. Food and Agriculture Organization of the United Nations (FAO). The State of World Fisheries and Aquaculture 2008. Part 1: World review of fisheries and aquaculture. Rome, Italy: FAO; 2009. URL Disponible en: ftp://ftp.fao.org/ docrep/fao/011/i0250e/i0250e01.pdf.

3. Chaiyapechara $S$, Casten $M$, Hardy $R$, Dong F. Fish performance, fillet characteristics, and health assessment index of rainbow trout (Oncorhynchus mykiss) fed diets containing adequate and high concentrations of lipid and vitamin E. Aquaculture 2003; 219:715-738.

4. Munasinghe $D$, Ichimaru $K$, Ryuno $M$, Ueki N, Matsui T, Sugamoto K, Kawahara $\mathrm{S}$, Sakai T. Lipid peroxidation-derived hepatotoxic aldehydes, 4-hydroxy-2E -hexenal in smoked fish meat products. Fish Sci 2003; 69:189-194.

5. Tocher D, Mourente G, Van Der Eecken A, Evjemo JO, Diaz E, Bell JG, et al. Effects of dietary vitamin $E$ on antioxidant defense mechanisms of juvenile turbot (Scophthalmus maximus L.), halibut (Hippoglossus hippoglossus L.) and sea bream (Sparus aurata L.). Aquac Nutr 2002; 8:195-207.

6. Association of official analytical chemist (AOAC). Official Methods of Analysis of the Association of Analytical Chemist, $16 a$ Edition. Washington DC: AOAC;1998.
7. Gunnlaugsdottir H, Ackman R. Three extraction methods for determination of lipids in fish meal: Evaluation of a hexane/ isopropanol method as an alternative to chloroform-based methods. J Sci Food Agric 1993; 61(2):235-240.

8. Instituto Colombiano de Normas Técnicas (ICONTEC). Norma Técnica Colombiana NTC 236: Grasas y aceites vegetales y animales. Determinación del índice de peróxido. Bogotá, Colombia: ICONTEC; 1998.

9. Instituto Colombiano de Normas Técnicas (ICONTEC). Norma Técnica Colombiana NTC 4197: Grasas y aceites vegetales y animales. Determinación del índice de anisidina. Bogotá, Colombia: ICONTEC; 2001.

10. Berglund AM, Sturve J, Förlin L, Nyholm NEI. Oxidative stress in pied flycatcher (Ficedula hypoleuca) nestlings from metal contaminated environments in northern Sweden. Environmental Research 2007; 105:330-339.

11. Lushchak V, Lushchak L, Mota A, HermesLima M. Oxidative stress and antioxidant defenses in goldfish Carassius auratus during anoxia and reoxygenation. Am J Physiol 2000; 280:R100-R107.

12. Ahmad I, Hamid T, Fatima $M$, Chand $H$, Jain S, Athar $M$ et al. Induction of hepatic antioxidants in freshwater catfish (Channa punctatus Bloch) is a biomarker of paper mill effluent exposure. Biochim Biophys Acta 2000; 1523:37-48.

13. Trenzado C, Hidalgo M, García-Gallego $M$, Morales A, Furné M, Domezain J, et al. Antioxidant enzymes and lipid peroxidation in sturgeon Acipenser naccarii and trout Oncorhynchus mykiss. A comparative study. Aquaculture 2006; 254:758-767. 
14. SAS/STAT: Guide for personal computer [programa de ordenador]. Versión 9.0.0. Cary (NC): SAS Institute Corporation; 2002.

15. Masson LS. Criterio de calidad para materias grasas utilizadas frecuentemente en la nutrición animal y de peces. Control de calidad se insumos y dietas acuícola. Documento de campo No 16. 1994; [En línea]. [Accesado 22 Jul 2010]. URL disponible en: http:// www.fao.org/docrep/field/003/AB482S/ AB482S10.htm.

16. Puangkaew J, Kiron V, Satoh S, Watanabe T. Antioxidant defense of rainbow trout (Oncorhynchus mykiss) in relation to dietary $n-3$ highly unsaturated fatty acids and vitamin $\mathrm{E}$ contents. Comp Biochem Physiol C Toxicol Pharmacol 2005; 140:187-196.

17. Mourente G, Diaz-Salvago E, Bell J, Tocher D. Increased activities of hepatic antioxidant defence enzymes in juvenile gilthead sea bream (Sparus aurata L.) fed dietary oxidised oil: attenuation by dietary vitamin E. Aquaculture 2002; 214: 343-361

18. Wang W, Wang A, Wang Y. Effect of supplemental L-ascorbyl-2polyphosphate in enriched live food on the antioxidant defense system of Penaeus vannamei of different sizes exposed to ammonia-N. Aquac Nutr 2006; 12:348-352.
19. Ansaldo M, Sacristán H, Wider E. Does starvation influence the antioxidant status of the digestive gland of Nacella concinna in experimental conditions? Comp Biochem Physiol C Toxicol Pharmcol 2007; 146:118-123.

20. Morales $A$, Pérez-Jiménez $A$, Hidalgo M, Abellán E, Cardenete G. Oxidative stress and antioxidant defenses after prolonged starvation in Dentex dentex liver. Comp Biochem Physiol C Toxicol Pharmcol 2004; 139: 153-161.

21. Marcon J, Wilhelm D. Antioxidant processes of the wild tambaqui, Colossoma macropomum (Osteichthyes, Serrasalmidae) from the Amazon. Comp Biochem Physiol C Toxicol Pharmcol 1999; 123:257-263.

22. Tort $M$, Hurley $D$, Fernández-Cobas C, Wooster G, Bowser P. Effects of Hydrogen Peroxide Treatments on Catalase and Glutathione Activity in Walleye Sander vitreus. J World Aquac Soc 2005; 36(4):577-586.

23. Furné $M$, García-Gallego $M$, Hidalgo $M$, Morales A, Domezain A, Domezain J et al. Oxidative stress parameters during starvation and refeeding periods in Adriatic sturgeon (Acipenser naccarii) and rainbow trout (Oncorhynchus mykiss). Aquac Nutr 2008; 15(6):587595. 